Positron range in PET imaging: an alternative approach for assessing and correcting the blurring

This article has been downloaded from IOPscience. Please scroll down to see the full text article.

2012 Phys. Med. Biol. 573931

(http://iopscience.iop.org/0031-9155/57/12/3931)

View the table of contents for this issue, or go to the journal homepage for more

Download details:

IP Address: 193.50.113.75

The article was downloaded on 30/05/2012 at 18:43

Please note that terms and conditions apply. 


\title{
Positron range in PET imaging: an alternative approach for assessing and correcting the blurring
}

\author{
L Jødal $^{1}$, C Le Loirec ${ }^{2}$ and C Champion ${ }^{3}$ \\ ${ }^{1}$ Department of Nuclear Medicine, Aalborg University Hospital, Denmark \\ ${ }^{2}$ Laboratoire Modélisation, Simulation et Systèmes, DRT/LIST/DCSI/LM2S, CEA Saclay, \\ Gif-sur-Yvette, France \\ ${ }^{3}$ Laboratoire de Physique Moléculaire et des Collisions, Université Paul Verlaine-Metz, France \\ E-mail: lajo@rn.dk
}

Received 19 December 2011, in final form 12 April 2012

Published 30 May 2012

Online at stacks.iop.org/PMB/57/3931

\begin{abstract}
Positron range impairs resolution in PET imaging, especially for high-energy emitters and for small-animal PET. De-blurring in image reconstruction is possible if the blurring distribution is known. Furthermore, the percentage of annihilation events within a given distance from the point of positron emission is relevant for assessing statistical noise. This paper aims to determine the positron range distribution relevant for blurring for seven medically relevant PET isotopes, ${ }^{18} \mathrm{~F},{ }^{11} \mathrm{C},{ }^{13} \mathrm{~N},{ }^{15} \mathrm{O},{ }^{68} \mathrm{Ga},{ }^{62} \mathrm{Cu}$ and ${ }^{82} \mathrm{Rb}$, and derive empirical formulas for the distributions. This paper focuses on allowed-decay isotopes. It is argued that blurring at the detection level should not be described by the positron range $r$, but instead the 2D projected distance $\delta$ (equal to the closest distance between decay and line of response). To determine these $2 \mathrm{D}$ distributions, results from a dedicated positron track-structure Monte Carlo code, Electron and POsitron TRANsport (EPOTRAN), were used. Materials other than water were studied with PENELOPE. The radial cumulative probability distribution $G_{2 \mathrm{D}}(\delta)$ and the radial probability density distribution $g_{2 \mathrm{D}}(\delta)$ were determined. $G_{2 \mathrm{D}}(\delta)$ could be approximated by the empirical function $1-\exp \left(-A \delta^{2}-B \delta\right)$, where $A=0.0266\left(E_{\text {mean }}\right)^{-1.716}$ and $B=0.1119\left(E_{\text {mean }}\right)^{-1.934}$, with $E_{\text {mean }}$ being the mean positron energy in $\mathrm{MeV}$ and $\delta$ in $\mathrm{mm}$. The radial density distribution $g_{2 \mathrm{D}}(\delta)$ could be approximated by differentiation of $G_{2 \mathrm{D}}(\delta)$. Distributions in other media were very similar to water. The positron range is important for improved resolution in PET imaging. Relevant distributions for the positron range have been derived for seven isotopes. Distributions for other alloweddecay isotopes may be estimated with the above formulas.
\end{abstract}




\section{Introduction}

Because of the improving spatial resolution of the physical detectors, the distance from positron emission to positron annihilation (positron range) is becoming a factor of increasing importance for the spatial resolution of positron emission tomography (PET). The positron range introduces uncertainty in the position of the positron emitter, resulting in blurring of the PET images. This effect is very clearly seen for high-energy emitters such as ${ }^{82} \mathrm{Rb}$, and also for less energetic emitters in small-animal PET imaging where blurring on a millimetre scale is important.

While the positron range in itself is a fundamental physical aspect of the slowing-down processes leading to annihilation, it is possible to correct for the blurring in the reconstructed images, if the reconstruction algorithm incorporates knowledge of the spatial distribution of the blurring effect (Cal-González et al 2009, Rahmim et al 2008, Ruangma et al 2006). Therefore, the distribution of positron ranges and its effect on PET imaging are subjects of prime importance, especially as technical advances may further improve detector resolution.

A point source emitting positrons will give rise to a three-dimensional (3D) distribution of annihilation points, which will characterize the positron range effect in the reconstructed images. But since a PET scanner measures lines of response (LORs), not annihilation positions, the 3D distribution is hard to measure directly (this also holds for time-of-flight PET, which reduces the length of the LOR, but still does not determine a point position). To determine the positron range, Derenzo (1979) made a series of often-cited measurements using material of very low density, in an experimental set-up where only a narrow plane was measured at a time. This corresponds to projecting the 3D annihilation distribution onto a single line, giving the $1 \mathrm{D}$ representation often used in papers on the positron range, see e.g. (Blanco 2006, Champion and Le Loirec 2007, Levin and Hoffman 1999).

However, considering a single detector pair (rather than a full detector ring), the logical projection will be 2D: the detector pair measures in the plane orthogonal to the LOR defined by the two detectors, and the positron range influences not only axial resolution (1D) but also transversal resolution. Thus, for a reconstruction algorithm modelling a positron range at the level of LORs, the distribution of the 2D projection of annihilation points will be very relevant.

In any homogeneous medium and in the absence of a magnetic field, the situation is spherically symmetrical, and the mathematical information in the 3D, 1D and 2D distributions will be the same, but converting e.g. a $1 \mathrm{D}$ distribution to a $2 \mathrm{D}$ distribution is not a simple calculation. However, 2D projections seem to have been given only little attention in the literature. Derenzo (1979) computed curves for ${ }^{11} \mathrm{C},{ }^{68} \mathrm{Ga}$ and ${ }^{82} \mathrm{Rb}$ (from a ${ }^{82} \mathrm{Sr}$ source, which decays to ${ }^{82} \mathrm{Rb}$ by electron capture) showing the 'fraction-projected annihilations occurring within a circle of radius $\mathrm{R}$ ', i.e. the cumulated $2 \mathrm{D}$ probability distribution. Sánchez-Crespo et al (2004) investigated ${ }^{18} \mathrm{~F},{ }^{15} \mathrm{O}$ and ${ }^{82} \mathrm{Rb}$ by projecting the $3 \mathrm{D}$ distribution onto a plane and reported point spread functions (PSFs) of this projection along a line. And recently, Cal-González et al (2010) and (2011) have considered 2D representations of the annihilation distribution and compared them with other representations. But little other work seems to have been conducted on $2 \mathrm{D}$ representations of the annihilation distribution.

This work aims to determine in details the cumulative 2D distributions for a series of medically relevant PET isotopes by means of a previously published dedicated positron trackstructure Monte Carlo simulation (Champion and Le Loirec 2006, 2007). Furthermore, it is aimed to provide a systematic procedure for estimating the cumulative distribution for other allowed-decay PET isotopes given only the mean positron energy $E_{\text {mean }}$. (Let us note that beta decay is allowed if parity is unchanged and nuclear spin change is 0 or \pm 1 (Krane 1988), e.g. $1^{+} \rightarrow 0^{+}$as in the decay ${ }^{18} \mathrm{~F} \rightarrow{ }^{18} \mathrm{O}$.) The focus on allowed-decay PET isotopes is 
practical. The energy distributions can be described by known, analytical functions, and many of the medical PET isotopes decay by allowed decay. Besides, differential distributions will be provided by following the same methodology. The results from the present Monte Carlo code (EPOTRAN, Electron and POsitron TRANsport in liquid and gaseous water) are compared with results from the more common PENELOPE code, and the influence of biological media other than water is estimated.

\section{Material and methods}

\subsection{Geometry and notation}

The complete blurring of a point source in an actual measurement will depend not only on positron range but also on photon non-collinearity and the properties of the detection system (such as crystal size and depth of interaction). However, all these effects can be considered individually and afterwards combined (Levin and Hoffman 1999, Qi et al 1998, Rahmim et al 2008). In the present context, we will assume point-like detectors and $180^{\circ}$ relative angle of the two photons emitted from annihilations, i.e. we focus on the positron range.

Consider a point source located at $(0,0,0)$ in a Cartesian coordinate system. Assuming spherical symmetry, the volume density $f(x, y, z)$ of annihilatons is a function of the positron ranger: $f(x, y, z)=f(r)$, where $r=\sqrt{x^{2}+y^{2}+z^{2}}$. Following the notation of Cal-González et al (2010), we will define the radial density as $g_{3 \mathrm{D}}(r)=4 \pi r^{2} f(r)=4 \pi r^{2} f(x, y, z)$.

The LORs measured parallel to a given direction will be cylindrically symmetrically distributed. As the distribution of LORs will be the same for all directions, we can treat the situation as if all LORs were parallel to the same direction. Taking the $z$-axis as the (arbitrary) choice of direction, the projection is onto the $x y$-plane. The distance from origin in 2D, $\delta=\sqrt{x^{2}+y^{2}}$, corresponds to the distance between the point source and the LOR. The 2D density distribution $f(x, y)$ is a function of these distances, $f(x, y)=f(\delta)$, with radial density $g_{2 \mathrm{D}}(\delta)=2 \pi \delta f(\delta)$.

The cumulative probability distribution $G_{2 \mathrm{D}}(\delta)$, i.e. the probability that a LOR will have a distance from the point source no greater than $\delta$, can be calculated by integration:

$$
G_{2 \mathrm{D}}(\delta)=\int_{0}^{\delta} g_{2 \mathrm{D}}\left(\delta^{\prime}\right) \mathrm{d} \delta^{\prime}
$$

Our notation for $g_{2 \mathrm{D}}$ and $G_{2 \mathrm{D}}$ is consistent with that of Cal-González et al (2010), except that we have chosen letter $\delta$ for the 2D radius (LOR distances from point source), reserving $r$ for the $3 \mathrm{D}$ radius (positron range).

\subsection{Positron energy spectra and Monte Carlo simulation}

The following series of medically relevant PET isotopes were considered: ${ }^{18} \mathrm{~F},{ }^{11} \mathrm{C},{ }^{13} \mathrm{~N},{ }^{15} \mathrm{O}$, ${ }^{68} \mathrm{Ga},{ }^{62} \mathrm{Cu}$ and ${ }^{82} \mathrm{Rb}$. These all are allowed-decay isotopes whose energetic disintegration spectra are represented by (Venkataramaiah et al 1985)

$$
N(E) \mathrm{d} E=C \cdot F(Z, E) \cdot p \cdot E \cdot\left(E_{\max }-E\right) \mathrm{d} E,
$$

with $E$ and $E_{\max }$ being the energy and maximal energy of $\beta$ particles, respectively, $p$ the corresponding momentum and $C$ a normalization constant. $F(Z, E)$ represents the Fermi factor used for taking into account the effect of the Coulomb field on the electron distribution.

The 3D distributions $f(x, y, z)$ of annihilation points were determined by using the fulldifferential Monte Carlo track-structure code called EPOTRAN developed by Champion and Le Loirec to study the decay of positron emitters of medical interest (Champion and Le 
Loirec 2007, Le Loirec and Champion 2007). In brief, for each isotope considered, a large number (about 250000 ) of positron tracks were simulated until annihilation, including all the processes, namely ionization, excitation, elastic scattering and positronium (Ps) formation. For more details, we refer the interested reader to (Champion et al 2012). The distributions $g_{2 \mathrm{D}}(\delta)$ were determined by binning $\delta=\sqrt{x^{2}+y^{2}}$ into $10 \mu \mathrm{m}$ bins. Finally, the cumulative probability $G_{2 \mathrm{D}}(\delta)$ was determined by numerical integration of $g_{2 \mathrm{D}}$.

\subsection{Generalization of distributions}

The resultant functions $G_{2 \mathrm{D}}(\delta)$ were compared among isotopes in two ways, namely either by plotting $G_{2 \mathrm{D}}(\delta)$ as a function of $\delta$ or by plotting $G_{2 \mathrm{D}}(\delta)$ as a function of $\delta / R_{\text {mean }}$, where $R_{\text {mean }}$ is the mean range of positrons for the isotope under consideration:

$$
R_{\text {mean }}=\iiint r \cdot f(x, y, z) \mathrm{d} x \mathrm{~d} y \mathrm{~d} z=\int_{0}^{\infty} r \cdot g_{3 \mathrm{D}}(r) \mathrm{d} r .
$$

An empirical formula for $G_{2 \mathrm{D}}(\delta)$ was then derived for each allowed-decay isotope, based on two adjustable parameters. The dependence of the parameters on mean positron energy $E_{\text {mean }}$ was also investigated. To estimate differential distributions $g_{2 \mathrm{D}}(\delta)$, the empirical formula for $G_{2 \mathrm{D}}(\delta)$ was differentiated, and the results compared with the distributions of $g_{2 \mathrm{D}}(\delta)$ which were determined from the Monte Carlo data.

\subsection{Effects of variation in biological tissues}

For a medium of a given composition, the range and thereby $\delta$ will scale inversely with density. But the shape of the positron range distribution may change with different composition (Lehnert et al 2011).

Presently, EPOTRAN is not able to simulate a positron track structure in a biological medium other than water. We have thus used the Monte Carlo code PENELOPE (2006 release) (Salvat et al 2006) first to compare the density-scaled range of positrons in biologically relevant materials with the range in water and secondly to study the decay of ${ }^{18} \mathrm{~F}$ in water, lung and bone.

PENELOPE is a Monte Carlo code of general purpose that permits us to simulate the coupled transport of photons, electrons and positrons in matter with a very good accuracy at low energies. Photons are transported within the conventional detailed method, whereas electrons and positrons are simulated using a mixed scheme where interactions are classified into hard and soft. Hard events are simulated step by step and involve angular deflection or energy losses above user-defined cutoffs, whereas soft events occurring between two hard collisions are simulated by means of an artificial single event. Simulation is controlled by seven parameters, fixed for the various materials used in the geometry:

(i) The absorption energy $E_{\mathrm{abs}}$ is the energy at which the track evolution is stopped. The kinetic energy of the particle is then locally deposited.

(ii) $C_{1}$ is the average angular deflection between two hard elastic collisions.

(iii) $C_{2}$ is the maximal fractional energy loss between two hard elastic collisions.

(iv) $W_{\mathrm{CC}}$ is the cutoff energy for hard inelastic interactions.

(v) $W_{\mathrm{CR}}$ is the cutoff energy for hard bremsstrahlung emission.

To be as close as possible as the simulations performed with EPOTRAN, we have chosen for water, lung and bone the same set of parameters which corresponds to a detailed simulation; thus $E_{\mathrm{abs}}=100 \mathrm{keV}$ for photons, electrons and positrons and $C_{1}, C_{2}, W_{\mathrm{CC}}$ and $W_{\mathrm{CR}}$ are equal to zero. 
Table 1. Mean and maximum values for energy and range for the emitted positrons (Champion and Le Loirec 2007, Le Loirec and Champion 2007).

\begin{tabular}{llllll}
\hline Isotopes & $E_{\text {mean }}(\mathrm{keV})$ & $E_{\max }(\mathrm{keV})$ & $R_{\text {mean }}(\mathrm{mm})$ & $\begin{array}{l}\text { Literature } \\
R_{\text {mean }}(\mathrm{mm})^{\mathrm{a}}\end{array}$ & $R_{\max }(\mathrm{mm})$ \\
\hline${ }^{18} \mathrm{~F}$ & 252 & 635 & 0.660 & 0.6 & 2.633 \\
${ }^{11} \mathrm{C}$ & 390 & 970 & 1.266 & 1.1 & 4.456 \\
${ }^{13} \mathrm{~N}$ & 488 & 1190 & 1.730 & 1.5 & 5.572 \\
${ }^{15} \mathrm{O}$ & 730 & 1720 & 2.965 & 2.5 & 9.132 \\
${ }^{68} \mathrm{Ga}$ & 844 & 1899 & 3.559 & 2.9 & 10.273 \\
${ }^{62} \mathrm{Cu}$ & 1280.6 & 2926 & 6.077 & & 16.149 \\
${ }^{82} \mathrm{Rb}$ & 1551 & 3378 & 7.491 & 5.9 & 18.603 \\
\hline \multicolumn{5}{c}{ a (Partridge et al 2006) } &
\end{tabular}

Tracking particles in PENELOPE is not obvious. It is however possible to determine the point of creation of particles, and in our case of annihilation photons. We have thus used a geometry consisting in concentric cylinders separated by $10 \mu \mathrm{m}$, which is the bin used for the distribution $g_{2 \mathrm{D}}(\delta)$. The cylinders are centred on $(0,0,0)$ which is the point of emission of positrons and they are full of water, bone or lung depending on the tissue under investigation. The simulation runs until the photons are detected on a spherical phase-space file which stores a lot of information concerning the particles and especially the body (the cylinder in our case) where they have been created. Thanks to these data, we can thus determine directly the 2D distribution of annihilation points and obtain the cumulative probability $G_{2 \mathrm{D}}(\delta)$.

\section{Results}

\subsection{Ranges and cumulative probability distributions}

The investigated radio-isotopes are listed in table 1 along with the mean and maximum values for energy and range for the emitted positrons (for more details see Champion and Le Loirec (2007) and Le Loirec and Champion (2007)). Compared to other results from the literature (see table 1 and figure 1), we obtain somewhat larger values for $R_{\text {mean }}$. This difference is due to the inclusion of Ps formation in the simulation, as the electrically neutral Ps loses energy more slowly than the charged $e^{+}$particle (Champion 2003, Champion and Le Loirec 2006).

The literature results for $R_{\text {mean }}$ do not include experimental data. Experimental results on $R_{\text {mean }}$ are, unfortunately, scarce. Cho et al (1975) measured a line source and determined an 'effective range' from the FW(0.1)M on this distribution, and Derenzo (1979), (1986) determined the root-mean-square spread for the $1 \mathrm{D}$ distribution. While such data can be used as indicators for $R_{\text {mean }}$, we are not aware of experimental results that aim at determining $R_{\text {mean }}$ as such.

It should be mentioned that while the Monte Carlo code used here assumes the neutral Ps to lose its kinetic energy mainly through phonon interactions at all energies, recent experimental results (Brawley et al 2010a, 2010b) found unexpectedly large Ps interactions at sub-keV energies. While these results are in themselves very interesting, a discrepancy in Ps cross section at very low energy will only have marginal influence on our results, since most of the path travelled by Ps will be with much higher kinetic energy.

The calculated cumulative probabilities $G_{2 \mathrm{D}}(\delta)$ for the closest distance $\delta$ are shown in figure 2. Not surprisingly, the distance $\delta$ that includes a given fraction of the LORs depends on the energy spectrum of the emitted positrons. However, when the same data are plotted as a function of $\delta / R_{\text {mean }}$, i.e. scaled by the isotope-dependent mean range, the results are rather similar among isotopes (see figure 3 ). 


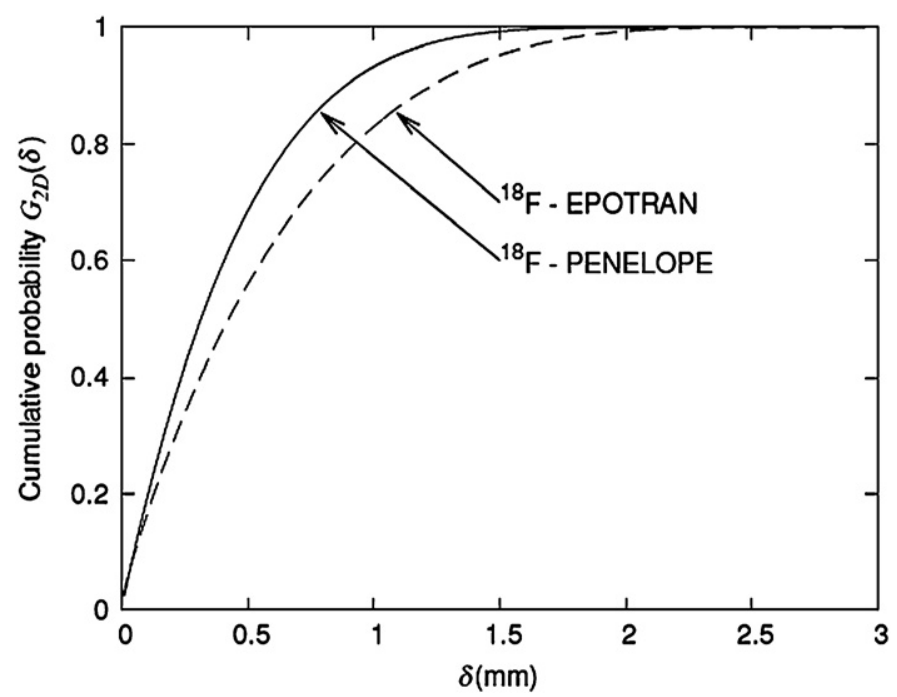

Figure 1. Cumulative probabilities for ${ }^{18} \mathrm{~F}$ obtained with EPOTRAN and PENELOPE.

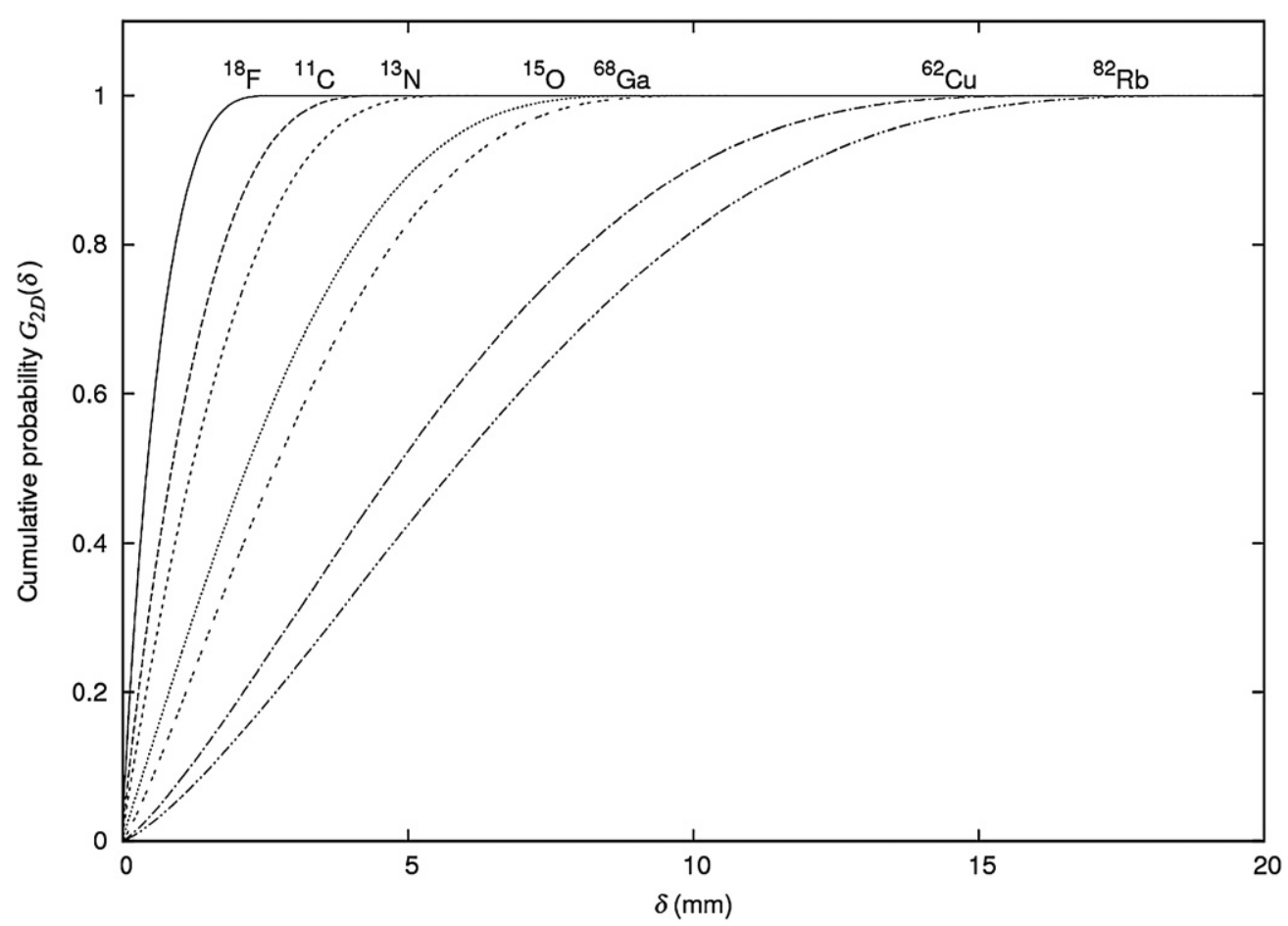

Figure 2. Cumulative probabilities $G_{2 \mathrm{D}}(\delta)$ obtained for the radio-isotopes investigated. 


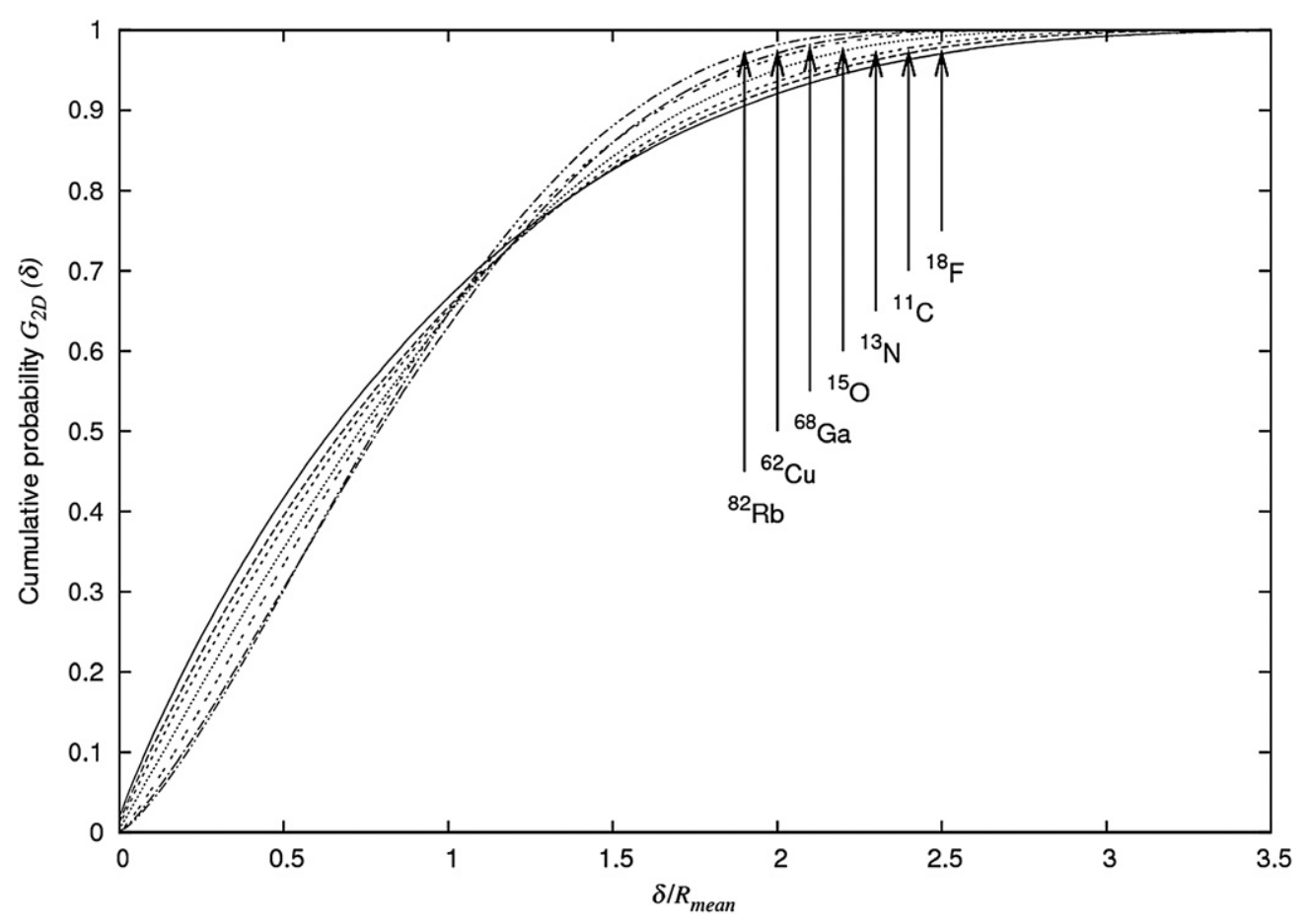

Figure 3. Cumulative probabilities $G_{2 \mathrm{D}}(\delta)$ versus $\delta / R_{\text {mean }}$.

\subsection{Empirical estimation of cumulative probability distributions}

Despite their similarity, the plots in figure 3 cannot be described by a single curve. Instead, we have for each isotope fitted the cumulative probability curves reported in figure 2 by the following empirical function:

$$
\zeta(\delta)=1-\exp \left(-A \delta^{2}-B \delta\right)
$$

This form is chosen such that for any positive values of $A$ and $B$, it will correctly rise monotonously from $\zeta(0)=0$ towards $1, \zeta(\infty)=1$. Furthermore, for any probability $0 \leqslant \zeta<1$ considered, the corresponding value of $\delta$ can be found as the positive solution to this equation:

$$
A \delta^{2}+B \delta+\ln (1-\zeta)=0
$$

The values obtained for the parameters $A$ and $B$ are reported in table 2.

The parameters $A$ and $B$ obviously depend on isotope. In figure 3, we scaled the curves by the isotope-dependent $R_{\text {mean }}$. For the parameters $A$ and $B$, we investigated instead dependence of the more easily determined value $E_{\text {mean }}$ and found that they could be described as power functions of $E_{\text {mean }}$ :

$$
A=0.0266\left(E_{\text {mean }}\right)^{-1.716} \text { and } B=0.1119\left(E_{\text {mean }}\right)^{-1.934},
$$

where $E_{\text {mean }}$ is given in $\mathrm{MeV}$, and the units of $A$ and $B$ are $\mathrm{mm}^{-2}$ and $\mathrm{mm}^{-1}$, respectively. The variations of $A$ and $B$ with $E_{\text {mean }}$ as well as the interpolated power functions are reported in figure 4.

As a simple check of these expressions, we also study the case of ${ }^{52} \mathrm{Fe}$, a radio-metal generator-produced isotope which emits positrons of relatively low energy, $E_{\max }=803.6 \mathrm{keV}$ 


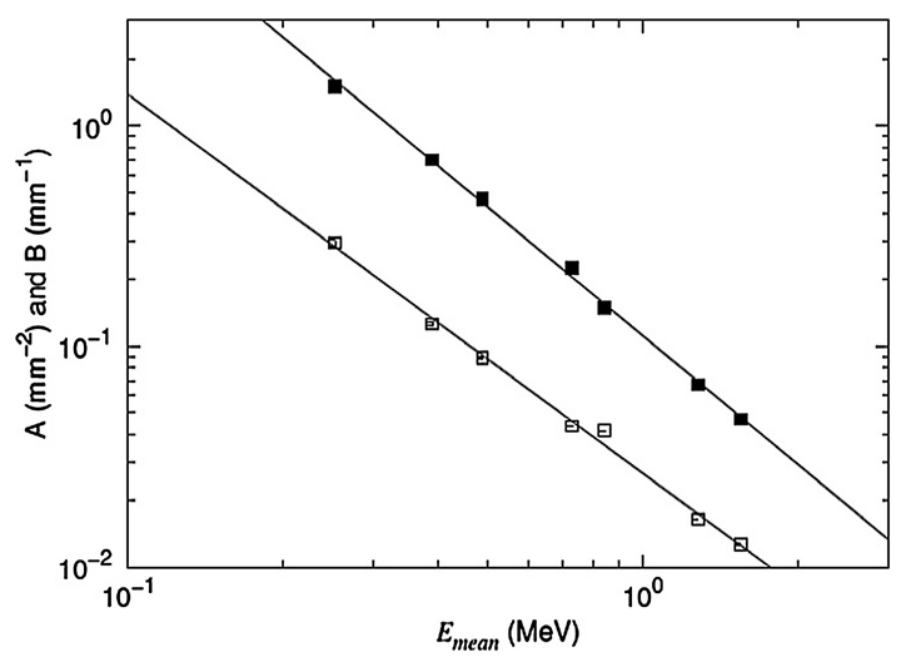

Figure 4. The fitting parameters $A$ and $B$ (open and solid squares, respectively) versus the mean energy of the isotope under consideration.

Table 2. Fitting parameters for the empirical cumulative probability given by (4). The standard errors are given in brackets for each isotope.

\begin{tabular}{lll}
\hline Isotopes & $A\left(\mathrm{~mm}^{-2}\right)$ & $B\left(\mathrm{~mm}^{-1}\right)$ \\
\hline${ }^{18} \mathrm{~F}$ & $0.2951( \pm 3.6 \%)$ & $1.5090( \pm 0.57 \%)$ \\
${ }^{11} \mathrm{C}$ & $0.1262( \pm 1.7 \%)$ & $0.7010( \pm 0.45 \%)$ \\
${ }^{13} \mathrm{~N}$ & $0.0885( \pm 1.0 \%)$ & $0.4681( \pm 0.38 \%)$ \\
${ }^{15} \mathrm{O}$ & $0.0436( \pm 0.54 \%)$ & $0.2266( \pm 0.33 \%)$ \\
${ }^{68} \mathrm{Ga}$ & $0.0414( \pm 0.35 \%)$ & $0.1499( \pm 0.34 \%)$ \\
${ }^{62} \mathrm{Cu}$ & $0.0164( \pm 0.28 \%)$ & $0.0671( \pm 0.40 \%)$ \\
${ }^{82} \mathrm{Rb}$ & $0.0126( \pm 0.23 \%)$ & $0.0468( \pm 0.42 \%)$ \\
\hline
\end{tabular}

and $E_{\text {mean }}=344 \mathrm{keV}=0.344 \mathrm{MeV}$. Clinically, ${ }^{52} \mathrm{Fe}$ has been used in haematological studies to follow iron kinetics, but such studies are complicated by the fact that ${ }^{52} \mathrm{Fe}$ decays to another PET isotope, ${ }^{52 \mathrm{~m}} \mathrm{Mn}$ (Haddad et al 2008, Lubberink et al 1999). By using (6), we have estimated the fitting parameters as $A=0.1660 \mathrm{~mm}^{-2}$ and $B=0.8813 \mathrm{~mm}^{-1}$. Figure 5 shows the calculated $G_{2 \mathrm{D}}(\delta)$ for ${ }^{52} \mathrm{Fe}$ along with the empirical function $\zeta(\delta)$ described by these values of $A$ and $B$.

\subsection{Radial probability density distributions}

For determination of PSFs, the probability density distribution should be known, either as the area density, $f(x, y)=f(\delta)$, or as the radial density, $g_{2 \mathrm{D}}(\delta)$. We here focus on the radial probability density, which can be compared to the result of differentiation of the estimate for cumulated probability, $\zeta(\delta)$ from (4):

$$
g_{2 \mathrm{D}}(\delta)=\frac{\mathrm{d}}{\mathrm{d} \delta} G_{2 \mathrm{D}} \approx \frac{\mathrm{d} \zeta}{\mathrm{d} \delta}=(2 A \delta+B) \cdot \exp \left(-A \delta^{2}-B \delta\right) .
$$

It will be noted that $\mathrm{d} \zeta / \mathrm{d} \delta$ is normalized to total area 1 , since $\zeta(0)=0$ and $\zeta(\infty)=1$. The Monte Carlo data are compared with the result of (7) in figure 6. 


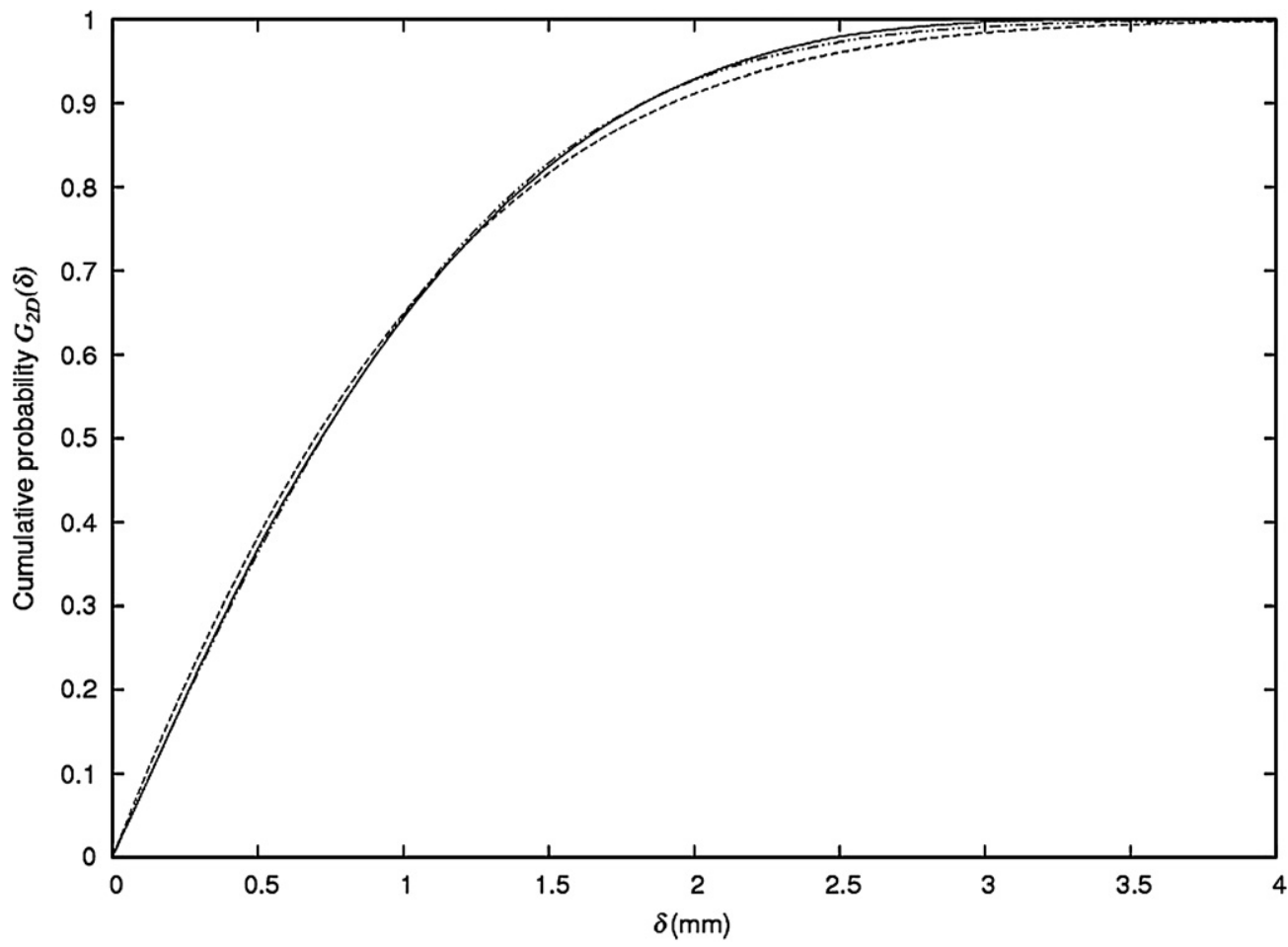

Figure 5. Calculated function $G_{2 \mathrm{D}}(\delta)$ along with the empirical function $\zeta(\delta)$ from estimated values of $A$ and $B$ for ${ }^{52} \mathrm{Fe}$ (solid and dashed line, respectively). A direct fit to the data is shown as a dot-dash-dot line almost coinciding with the full line.

Table 3. Approximate mean range for two isotopes (chosen to represent low and high energy) calculated with PENELOPE in the continuing slowing down approximation. For soft and lung tissue, the largest difference from water is $1.2 \%$ whereas it is up to $13 \%$ for bone tissue.

\begin{tabular}{lllll}
\hline \multicolumn{2}{l}{ Biological materials (ICRP compositions) } & & \multicolumn{2}{l}{ range $\cdot$ density $\left(\mathrm{g} \mathrm{cm}^{-2}\right)$} \\
\cline { 1 - 2 } Name & Density $\rho\left(\mathrm{g} \mathrm{cm}^{-3}\right)$ & & ${ }^{18} \mathrm{~F}$ & ${ }^{62} \mathrm{Cu}$ \\
\hline Water & 1.00 & 0.0622 & 0.595 \\
Lung & 0.26 & 0.0634 & 0.597 \\
Adipose & 0.98 & 0.0613 & 0.591 \\
Muscle & 1.07 & 0.0635 & 0.602 \\
Bone & 1.92 & 0.0702 & 0.666 \\
\hline
\end{tabular}

\subsection{Effects of variation in biological tissues}

The PENELOPE results for the density-scaled positron range in different media are given in table 3 . It is seen that soft tissue differs very little from water, while bone tissue deviates by about $13 \%$.

In figure 7, we have reported the cumulative 2D probabilities obtained in PENELOPE for ${ }^{18} \mathrm{~F}$ in water, bone and lung. The data are plotted as a function of $\delta \cdot \rho$, with $\rho$ being the density of the biological tissue. The tendency for bone data to have larger distances can be seen as 

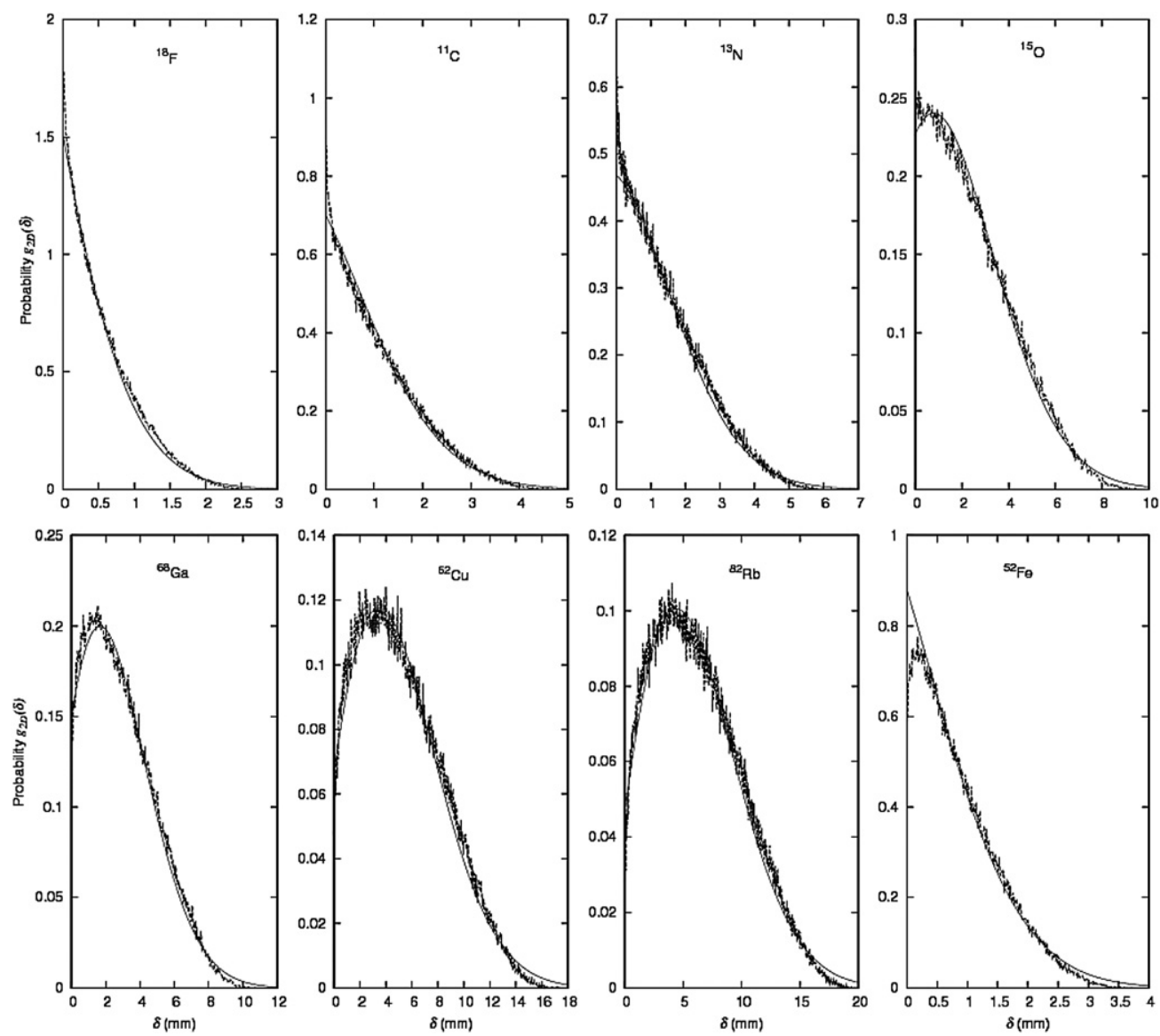

Figure 6. Functions $g_{2 \mathrm{D}}(\delta)$ taken from Monte Carlo calculations (broken lines) compared with the expression from (7) (smooth lines), using values of $A$ and $B$ from table 2. For ${ }^{52} \mathrm{Fe}$, the values of $A$ and $B$ from (6) were used. Monte Carlo data are reported with a $10 \mu \mathrm{m}$ bin for ${ }^{18} \mathrm{~F},{ }^{11} \mathrm{C},{ }^{13} \mathrm{~N}$ and ${ }^{52} \mathrm{Fe}$, and with a $30 \mu \mathrm{m}$ bin for ${ }^{15} \mathrm{O},{ }^{68} \mathrm{Ga},{ }^{62} \mathrm{Cu}$ and ${ }^{82} \mathrm{Rb}$.

the bone curve being slightly below the other two curves in the upper half of the figure, but overall the density-corrected distributions are very similar for all three media.

\section{Discussion}

\subsection{Cumulative and differential $2 D$ probability distributions}

As noted in the introduction, the choice of 2D distributions is very relevant when considering measurements by individual detector pairs. The cumulative distribution $G_{2 \mathrm{D}}(\delta)$ shows us that for all investigated isotopes, almost two-third of the annihilations take place within $\delta \leqslant$ $R_{\text {mean }}$ (see figure 3), meaning that $R_{\text {mean }}$ can be used as a rough estimate of the expected broadening due to the positron range.

When based on Monte Carlo data, rather than mathematical functions, the cumulative (integral) distribution is more robust than the differential distribution. But a reconstruction algorithm involving modelling of the positron range will typically need the differential 


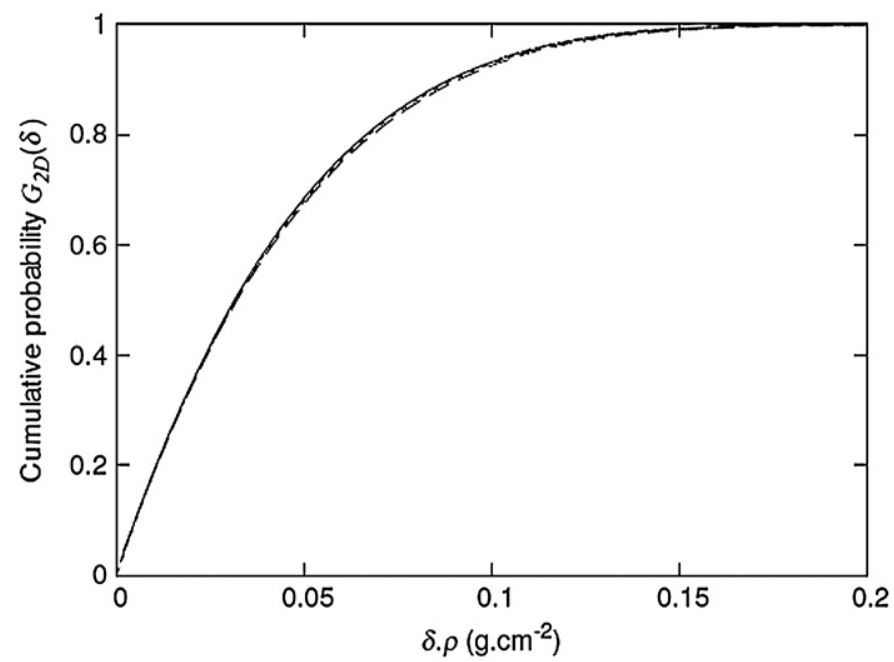

Figure 7. Cumulative probability for water (solid line), bone (dash line) and lung (dotted line) as a function of $\delta \cdot \rho$. All three curves are based on PENELOPE results.

distribution, either as area density $f(\delta)$ or radial density $g_{2 \mathrm{D}}(\delta)$. As demonstrated in figure 6 , the radial distributions can be well approximated by using (7) with the values of $A$ and $B$ from table 2. If $A$ and $B$ are determined from the simple, empirical relations (6), then the distribution will of course be less precise, but the curves for ${ }^{52} \mathrm{Fe}$ in figures 5 and 6 indicate that such distributions can be used as the first approximation for other allowed-decay isotopes than those in table 2.

Our focus on the radial density has the advantage of avoiding the very peaked (cuspshaped) distributions found for both 1D (Derenzo 1979) and 2D (Sánchez-Crespo et al 2004) non-radial distributions. These peaks are so sharp that the peak height becomes dependent on the details of the calculations (e.g., bin size), meaning that measures dependent on peak height (such as FWHM) do not have a strict meaning (Levin and Hoffman 1999).

In fact, our own results indicate that the radial distribution $g_{2 \mathrm{D}}(\delta)$ does not necessarily go to zero for $\delta \rightarrow 0$ (see figure 6), i.e. the area distribution $f(\delta)=g_{2 \mathrm{D}}(\delta) /(2 \pi \delta)$ may diverge for $\delta \rightarrow 0$, in which case FWHM is meaningless. Note that such a divergence is not unphysical as long as the area under the singularity is finite. For example, a simple harmonic oscillator, $x=$ $A \sin (\omega t)$, will have a density distribution for $x$ which diverges at $x= \pm A$, because velocity is zero in these positions. In our case, $g_{2 \mathrm{D}}$ can be integrated into $G_{2 \mathrm{D}}$, with the (physically correct) implication that only a finite number of positrons will be detected near $\delta=0$.

It can be argued against the form (4) for the cumulative distribution $G_{2 \mathrm{D}}(\delta)_{\text {estimate }}=$ $\zeta(\delta)$ that it never reaches 1 , and therefore the differential distributions $g_{2 \mathrm{D}}(\delta)_{\text {estimate }}=\mathrm{d} \zeta / \mathrm{d} \delta$ never fall to 0 , despite positrons having a maximum range. The effect of this can be seen in figure 6, where the final part of the curves $g_{2 \mathrm{D}}(\delta)_{\text {estimate }}$ tend to be slightly above the Monte Carlo data, especially for the high-energy isotopes. Similar objections could however be raised against describing positron range distributions by exponentials (Derenzo 1979) or Gaussian functions (Palmer and Brownell 1992). Mathematically, it can be seen that $\mathrm{d} \zeta / \mathrm{d} \delta$ will eventually approach the zero value faster than a Gaussian function: $\mathrm{d} \zeta / \mathrm{d} \delta$ can be considered as the product of a Gaussian, $\exp \left(-A \delta^{2}\right)$, and a factor that goes towards zero for large distances, $(2 A \delta+B) \cdot \exp (-B \delta)$, possibly after an initial rise. If a cutoff distance $\delta_{\text {cutoff }}$ is introduced, 
setting $g_{2 \mathrm{D}}(\delta)_{\text {estimate }}=0$ for $\delta>\delta_{\text {cutoff }}$, then the area under the curve will then be slightly less than 1 , namely $\zeta\left(\delta_{\text {cutoff }}\right)$. A possible choice for $\delta_{\text {cutoff }}$ can be $R_{\max }$ which can be estimated from $E_{\text {mean }}$ as a linear function, based on table 1 (fit not shown).

In short, our formulation based on $A$ and $B$ is suitable for applications dependent on the whole distribution (such a de-blurring), while it is less suitable or unsuitable for applications sensitive to the behaviour of a small fraction of the positrons (such as the determination of maximal distances). Figure 7 indicates that the same values of $A$ and $B$ can be used for different media if distances are scaled by density, as has already been proposed by Lehnert et al (2011) for a $1 \mathrm{D}$ distribution model.

\subsection{Limitations}

The current study was made only for allowed-decay isotopes. Non-allowed-decay isotopes can have other relations between $E_{\text {mean }}$ and spectrum shape, meaning that the empirical relation (6) cannot be expected to give usable results for non-allowed-decay isotopes. As an example, we tried it for ${ }^{124} \mathrm{I}$ (data not shown), which is non-allowed decay, and found large discrepancies from expected $G_{2 \mathrm{D}}(\delta)$.

\section{Conclusion}

Nowadays, it is well known that when high-energy positron emitters are used in PET scanning, the positron range adds to resolution degradation. In the major part of existing studies, this degradation is related to the positron range $(r)$. In this work, we have proposed not to use the positron range but instead the shortest distance $(\delta)$ between the point of emission and the LOR. Annihilation point radial 2D density distributions as well as cumulated probabilities for a series of medically relevant PET isotopes were provided by means of Monte Carlo simulation. Empirical expressions of these distributions were then proposed for the allowed-decay PET isotopes investigated here.

These results allow an easy estimation of the percentage of annihilations occurring within a given distance and may give approximate density distributions for use in reconstruction algorithms taking the positron range into account.

\section{References}

Blanco A 2006 Positron range effects on the spatial resolution of RPC-PET IEEE Nucl. Sci. Symp. Conf. Rec. pp 2570-3

Brawley S J, Armitage S, Beale J, Leslie D E, Williams A I and Laricchia G 2010a Electron-like scattering of positronium Science 330789

Brawley S J, Williams A I, Shipman M and Laricchia G 2010b Resonant scattering of positronium in collision with $\mathrm{CO}_{2}$ Phys. Rev. Lett. 105263401

Cal-González J, Herraiz J L, España S, Corzo P M G and Udías J M 2011 A general framework to study positron range distributions IEEE Nucl. Sci. Symp. Conf. Rec. pp 2733-7

Cal-González J, Herraiz J L, España S, Desco M, Vaquero J J and Udías J M 2009 Positron range effects in high resolution 3D PET imaging IEEE Nucl. Sci. Symp. Conf. Rec. pp 2788-91

Cal-González J, Herraiz J L, España S, Desco M, Vaquero J J and Udías J M 2010 Validation of PeneloPET positron range estimations IEEE Nucl. Sci. Symp. Conf. Rec. pp 2396-9

Champion C 2003 Theoretical cross sections for electron collisions in water: structure of electron tracks Phys. Med. Biol. 48 2147-68

Champion C and Le Loirec C 2006 Positron follow-up in liquid water: I. A new Monte Carlo track-structure code Phys. Med. Biol. 51 1707-23

Champion C and Le Loirec C 2007 Positron follow-up in liquid water: II. Spatial and energetic study for the most important radioisotopes used in PET Phys. Med. Biol. 52 6605-25 
Champion C, Le L C and Stosic B 2012 EPOTRAN: a full-differential Monte Carlo code for electron and positron transport in liquid and gaseous water Int. J. Radiat. Biol. 88 54-61

Cho Z H, Chan J K, Ericksson L, Singh M, Graham S, MacDonald N S and Yano Y 1975 Positron ranges obtained from biomedically important positron-emitting radionuclides J. Nucl. Med. 16 1174-6

Derenzo S E 1979 Precision measurement of annihilation point spread distributions for medically important positron emitters Positron Annihilation ed R R Hasiguti and K Fuliwara (Sendai, Japan: The Japan Institute of Metals) pp 819-23

Derenzo S E 1986 Mathematical removal of positron range blurring in high-resolution tomography IEEE Trans. Nucl. Sci. 33 565-9

Haddad F, Ferrer L, Guertin A, Carlier T, Michel N, Barbet J and Chatal J F 2008 ARRONAX, a high-energy and high-intensity cyclotron for nuclear medicine Eur. J. Nucl. Med. Mol. Imaging 35 1377-87

Krane K S 1988 Introductory Nuclear Physics (New York: Wiley)

Le Loirec C and Champion C 2007 Track structure simulation for positron emitters of medical interest: part I. The case of the allowed decay isotopes Nucl. Instrum. Methods Phys. Res. A 582 644-53

Lehnert W, Gregoire M C, Reilhac A and Meikle S R 2011 Analytical positron range modelling in heterogeneous media for PET Monte Carlo simulation Phys. Med. Biol. 56 3313-35

Levin C S and Hoffman E J 1999 Calculation of positron range and its effect on the fundamental limit of positron emission tomography system spatial resolution Phys. Med. Biol. 44 781-99

Lubberink M, Tolmachev V, Beshara S and Lundqvist H 1999 Quantification aspects of patient studies with Fe-52 in positron emission tomography Appl. Radiat. Isot. 51 707-15

Palmer M R and Brownell G L 1992 Annihilation density distribution calculations for medically important positron emitters IEEE Trans. Med. Imaging $11373-8$

Partridge M, Spinelli A, Ryder W and Hindorf C 2006 The effect of $\beta^{+}$energy on performance of a small animal PET camera Nucl. Instrum. Methods Phys. Res. A 568 933-6

Qi J Y, Leahy R M, Cherry S R, Chatziioannou A and Farquhar T H 1998 High-resolution 3D Bayesian image reconstruction using the microPET small-animal scanner Phys. Med. Biol. 43 1001-13

Rahmim A, Tang J, Lodge M A, Lashkari S, Ay M R, Lautamaki R, Tsui B M W and Bengel F M 2008 Analytic system matrix resolution modeling in PET: an application to Rb-82 cardiac imaging Phys. Med. Biol. 53 5947-65

Ruangma A, Bai B, Lewis J S, Sun X K, Welch M J, Leahy R and Laforest R 2006 Three-dimensional maximum a posteriori (MAP) imaging with radiopharmaceuticals labeled with three $\mathrm{Cu}$ radionuclides $\mathrm{Nucl}$. Med. Biol. 33 217-26

Salvat F, Fernández-Varea J M and Sempau J 2006 PENELOPE-A code system for Monte Carlo simulation of electron and photon transport (Issy-les-Moulineaux: OECD Nuclear Energy Agency)

Sánchez-Crespo A, Andreo P and Larsson S A 2004 Positron flight in human tissues and its influence on PET image spatial resolution Eur. J. Nucl. Med. Mol. Imaging 31 44-51

Venkataramaiah P, Gopala K, Basavaraju A, Suryanarayana S S and Sanjeeviah H 1985 A simple relation for the Fermi function J. Phys. G: Nucl. Part. Phys. 11 359-64 